\title{
Complementary inhibition of cerebral aneurysm formation by eNOS and nNOS
}

\author{
Tomohiro Aoki ${ }^{1,2}$, Masaki Nishimura ${ }^{1}$, Hiroharu Kataoka ${ }^{1}$, Ryota Ishibashi ${ }^{1}$, Kazuhiko Nozaki ${ }^{3}$ \\ and Susumu Miyamoto ${ }^{1}$
}

The rupture of cerebral aneurysm (CA) and subsequent subarachnoid hemorrhage can cause fatal results. Recent experimental findings have suggested that the mechanism of CA formation is based on chronic inflammation in arterial walls by hemodynamic force. Endothelial nitric oxide synthase (eNOS) protects arterial walls from vascular inflammation by relieving hemodynamic force through nitric oxide (NO) production. Thus, the expression and protective role of eNOS in CA formation have been investigated in this study. In this study, experimental induced rodent CA models by carotid ligation and systemic hypertension were used. The expression of eNOS was examined in rat CA models and revealed that it was decreased at the site of CA formation. Next, CA was induced in eNOS ${ }^{-1-}$ mice to clarify the role of eNOS in CA formation. In eNOS ${ }^{-1-}$ mice, the incidence of CA formation was similar to that found in wild-type mice. In CA walls of $\mathrm{eNOS}^{-1-}$ mice, the expression of neuronal nitric oxide synthase (nNOS) was upregulated compared with that in wild-type mice, suggesting the compensatory effect of nNOS. Hence, eNOS ${ }^{-1-} \mathrm{nNOS}^{-1-}$ mice were generated, underwent CA induction and confirmed that eNOS ${ }^{-1-} \mathrm{nNOS}^{-1-}$ mice exhibited an increased incidence of CA formation accompanied by accelerated macrophage infiltration. These results suggested that the deficiency of eNOS could be compensated by nNOS upregulation in cerebral arteries and that the eNOS and nNOS complementarily had the protective role in CA formation. The results of this study will provide us with new insight about the mechanisms of CA formation and the functional redundancy between eNOS and nNOS in cerebral arteries.

Laboratory Investigation (2011) 91, 619-626; doi:10.1038/labinvest.2010.204; published online 14 February 2011

KEYWORDS: cerebral aneurysm; compensation; eNOS; macrophage; nNOS

Subarachnoid hemorrhage is a life-threatening disease, and its mortality and morbidity are high despite the recent technical advancement. ${ }^{1}$ The main cause of subarachnoid hemorrhage is rupture of a pre-existing cerebral aneurysm (CA). Although CA is an important disease in the public health because of its high prevalence ${ }^{2}$ and the severity of subsequent subarachnoid hemorrhage, ${ }^{1,3}$ the specific mechanisms of CA formation are not fully understood. Given that CAs occur at the bifurcation site of cerebral arteries, where hemodynamic force is most strongly loaded, hemodynamic force is considered as an underlying condition of CA formation. ${ }^{4,5}$ In arterial walls, protective mechanisms against hemodynamic force are well recognized. ${ }^{6,7}$ Endothelial nitric oxide synthase (eNOS) is one of the most important molecules. ${ }^{6,8}$ It is an enzyme that catalyzes nitric oxide $(\mathrm{NO})$ to dilate arteries and reduces the hemodynamic stress to arterial walls. In various pathological conditions, eNOS has a protective role against disease formation and progression through the reduction of hemodynamic force loaded. ${ }^{9,10}$ For example, in abdominal aortic aneurysm (AAA), eNOS deficiency has resulted in the increased incidence of AAA formation. ${ }^{10}$ However, the role of eNOS in CA formation has not been well characterized. In this study, we examined the role of eNOS in CA formation using experimental CA models of rats and mice. ${ }^{11-13}$

\section{MATERIALS AND METHODS Induction of Experimentally Induced CAs in Rats}

Animal care and experiments complied with Japanese community standards on the care and use of laboratory animals, and all procedures of animal experiments were approved by the committee for animal experiments of Kyoto University.

\footnotetext{
${ }^{1}$ Department of Neurosurgery, Kyoto University Graduate School of Medicine, Kyoto, Japan; ${ }^{2}$ Department of Pharmacology, Kyoto University Graduate School of Medicine, Kyoto, Japan and ${ }^{3}$ Department of Neurosurgery, Shiga University of Medical Science, Shiga, Japan

Correspondence: Dr M Nishimura, MD, PhD, Department of Neurosurgery, Kyoto University Graduate School of Medicine, 54 Kawaharacho, Shogoin, Sakyo-ku, Kyoto, 606-8507, Japan.

E-mail: masakins@kuhp.kyoto-u.ac.jp

Received 26 April 2010; revised 16 November 2010; accepted 18 November 2010
} 
Rat CAs were induced as previously described ${ }^{13,14}$ (Supplementary Figure 1) in which we have repeatedly demonstrated the similarity of this model with human CAs and suitability as a CA model. ${ }^{11,15,16}$ Briefly, under the general anesthesia by pentobarbital $(50 \mathrm{mg} / \mathrm{kg}$ i.p.), the left carotid artery and posterior branches of the bilateral renal arteries were simultaneously ligated with 10-0 nylon in 7-week-old male Sprague-Dawley rats. Animals were fed high salt diet containing $8 \%$ sodium chloride and $0.12 \% \beta$-aminopropionitrile (BAPN: Tokyo Chemical Industry, Tokyo, Japan), an inhibitor of lysyl oxidase that catalyzed the crosslinking of collagen and elastin, from just after the surgical procedure. Lysyl oxidase was decreased in CA walls and contributed to CA formation. ${ }^{17}$ BAPN mimicked the decrease of lysyl oxidase in CA walls and was used for amplifying the degenerative changes of arterial walls and for increasing the incidence and enlargement of CA formation. ${ }^{17}$

Blood pressure was measured by tail-cuff methods without any anesthesia.

\section{Induction of Experimental CAs in Mice}

$\mathrm{eNOS}^{-1-}$ mice ${ }^{18}$ and neuronal nitric oxide synthase $(\text { nNOS })^{-1-}$ mice, whose genetic background was C57/B6, were purchased from Jackson Laboratory (Bar Harbor, ME, USA). eNOS ${ }^{+1-} \mathrm{NOS}^{-1-}$, $\mathrm{eNOS}^{-1-} \mathrm{nNOS}^{+-}$and $\mathrm{eNOS}^{-1-}$ $\mathrm{nNOS}^{-1-}$ mice were generated by mating eNOS ${ }^{-1-}$ mice and nNOS $^{-1-}$ mice. For the confirmation of knockout allele, genotyping using primers described in Jackson Laboratory Homepage was performed. Only male littermates were used in the experiment. At 7 weeks, CAs were induced as previously described. ${ }^{12}$ Briefly, the left carotid artery was ligated under the general anesthesia by Fluothane. After 1 week, posterior branches of the bilateral renal artery were ligated. Animals were fed the same food as used in rat CA model. We could not induce CAs in mice without BAPN, and hence we added this compound in food. Blood pressure was measured in all mice by tail-cuff method like rat.

After 5 months of CA induction, mice were killed and transcardinally perfused by $4 \%$ paraformaldehyde. Then, the entire circle of Willis was stripped from brain surface and the right anterior cerebral artery/olfactory artery (ACA/OA) bifurcation was dissected. Dissected ACA/OA bifurcation was embedded and frozen. Sections, $5-\mu \mathrm{m}$ thin, were cut and mounted on silane-coated slides. After Elastica van Gieson staining, CA formation at right ACA/OA bifurcation was assessed under a light microscope. Aneurysm refers to an outward bulging of the arterial wall detected by a light microscopy (Supplementary Figure 1). One of the pathological features of CA walls was the discontinuity of the internal elastic lamina and thinning of media. The degenerative change of the internal elastic lamina leads to the weakening of arterial stiffness and is considered as the underlying pathology of CA formation. ${ }^{19-21}$ Hence, we defined early aneurysmal change as a lesion with the discontinuity of the internal elastic lamina without apparent outward bulging of the arterial wall. Advanced aneurysm refers to an obvious outward bulging of the arterial wall with the fragmentation or disappearance of the internal elastic lamina. Three independent researchers assessed the histopathological changes.

\section{Immunohistochemistry}

At 1 or 3 months after CA induction, all rats were deeply anesthetized and perfused transcardinally with $4 \%$ paraformaldehyde. As a control sample, age-matched male Sprague-Dawley rats were killed as described above. ACA/OA bifurcation was stripped, embedded and frozen. Sections, $5-\mu \mathrm{m}$ thin, were cut and mounted on silane-coated slides. After blocking with 5\% Donkey serum, primary antibody was applied and incubated followed by the incubation with fluorescence labeled secondary antibodies (Jackson Immuno Research, Baltimore, MD, USA). Then, the slides were excited for fluorescence by illumination through a fluorescence microscope system (BX51N-34-FL-1, Olympus, Tokyo, Japan). As a negative control experiment, we performed the immunostaining without primary antibodies and identified the nonspecific signal.

The eNOS-positive area in $100 \mu \mathrm{m}^{2}$ around a dome of CA or corresponding site of control artery were calculated as the ratio of fluorescence-positive area for eNOS to arterial wall by Scion Image (Scion, Frederick, MD, USA).

The primary antibodies used were listed as follows: mouse polyclonal anti-CD68 antibody (HyCult, Uden, The Netherlands), rabbit polyclonal anti-eNOS antibody (Lab Vision, Fermont, CA, USA), mouse monoclonal anti-eNOS (Biomol Research Laboratories, Plymouth, PA, USA), rabbit polyclonal anti-nNOS antibody (Santa-Cruz, Santa Cruz, CA, USA), rabbit anti-inducible nitric oxide synthase (iNOS) antibody (Santa-Cruz) and goat polyclonal anti-platelet/ endothelial cell adhesion molecule (PECAM)-1 antibody (Santa-Cruz).

The number of infiltrated macrophages was calculated as the number of CD68-positive cells in $50 \mu \mathrm{m}^{2}$ around dome of CAs.

\section{Western Blotting}

At 1 or 3 months after CA induction, the whole-cell lysate from the anterior portion of the circle of Willis was extracted using Complete Lysis M Kit (Roche, Basel, Switzerland). Whole-cell lysate $(15 \mu \mathrm{g})$ was used in each experiment. We made the plural membranes with the same samples and same amount of proteins to avoid the stripping of probes. After electrophoresis, transfer to PDVF membrane (GE Healthcare, Buckinghamshire, UK) and blocking with ECL plus blocking agent (GE Healthcare), membranes were incubated with primary antibodies (mouse monoclonal anti-eNOS antibody (Biomol Research Laboratories), others are same in immunohistochemistry) followed by incubation with horseradish peroxidase-conjugated anti-IgG antibody (GE Healthcare). The signal was detected by chemiluminescent reagent (ECL plus Western Blotting Detection System, 
GE Healthcare). $\alpha$-Tubulin was served as an internal control. Densitometric analysis included five independent experiments.

\section{PCR Reactions in Mice}

Total RNA from the whole circle of Willis was extracted by RNeasy Fibrous Tissue Mini Kit (QIAGEN, Hilden, Germany) and converted into cDNA by Sensiscript reverse transcriptase (QIAGEN). PCR was performed using HotStar Taq polymerase (QIAGEN). $\beta$-Actin was used as an internal control. As a negative control experiment, we performed the experiment without primers. PCR products were separated by the electrophoresis in $2 \%$ agarose gel. Densitometric analysis included five samples per group.

The primer sets used were following: forward $5^{\prime}$-GCAC AGAAGTGAGGGTATGC-3' , reverse $5^{\prime}$-ACAAGGTCCGATT CAACAGC- $3^{\prime}$ for nNOS; forward $5^{\prime}$-CCTCTTTCAGGTCA CTTTGG- ${ }^{\prime}$, reverse $5^{\prime}$-GCTTGGGTCTTGTTCACTCC- ${ }^{\prime}$ for iNOS; forward $5^{\prime}$-TGAACCACTTCCATTCTTCG-3', reverse $5^{\prime}$-GCAGGCTCTCACCTACTTCC- $3^{\prime}$ for eNOS; forward $5^{\prime}$-GGAGCATCCACGTGTTGGC-3' , reverse 5'-ACAGCTTC TTTGGGACACC- $3^{\prime}$ for monocyte chemoattractant protein-1 (MCP-1); forward 5'-CTGCTCCACAGGATTTTGG-3', reverse $5^{\prime}$-TCCCCACCATTGAAGATACC-3' for vascular cell adhesion molecule-1 (VCAM-1); and forward 5'-CCCTAAGGCCAAC CGTGAAA- $3^{\prime}$, reverse $5^{\prime}$-ACGACCAGAGGCATACAGGGA-3 ${ }^{\prime}$ for $\beta$-actin.

\section{Statistical Analysis}

Data (mean (s.d.)) were analyzed by the Mann-Whitney $U$-test for a two-group comparison, and Kruskal-Wallis oneway ANOVA on ranks followed by the Turkey-Kramer test for a multiple comparison. The incidence of aneurysmal changes was analyzed using Fisher's exact test. Differences were considered statistically significant at $P<0.05$.

\section{RESULTS}

\section{Expression of eNOS in Experimentally Induced CAs in Rats}

First, to confirm the expression of eNOS during CA formation, immunostaining and western blot analysis for eNOS were performed in experimental CAs of rats.

In control arterial walls, immunostaining showed that eNOS was expressed mainly in endothelial cells and partially in the adventitia tissue (Figure 1b). In CA walls at 1 and 3 months after CA induction, eNOS was also expressed mainly in endothelial cells, and its expression in CA lesions was decreased compared with that in control arteries $(0$ month (M); 8.0\% (3.3), 1M; $2.9 \%$ (2.2), 3M; 0.9\% (0.9), $n=4$ for all groups, $0 \mathrm{M}$ vs $1 \mathrm{M}$ : $P=0.040,0 \mathrm{M}$ vs $3 \mathrm{M}: P=0.020$; Figure $1 \mathrm{~d}$ and f; CA walls are shown in square).

In western blot analysis using proteins from the circle of Willis, the expression of eNOS was significantly upregulated after CA formation ( 1 versus 3 months: $P<0.01, n=5$; Figure 2).

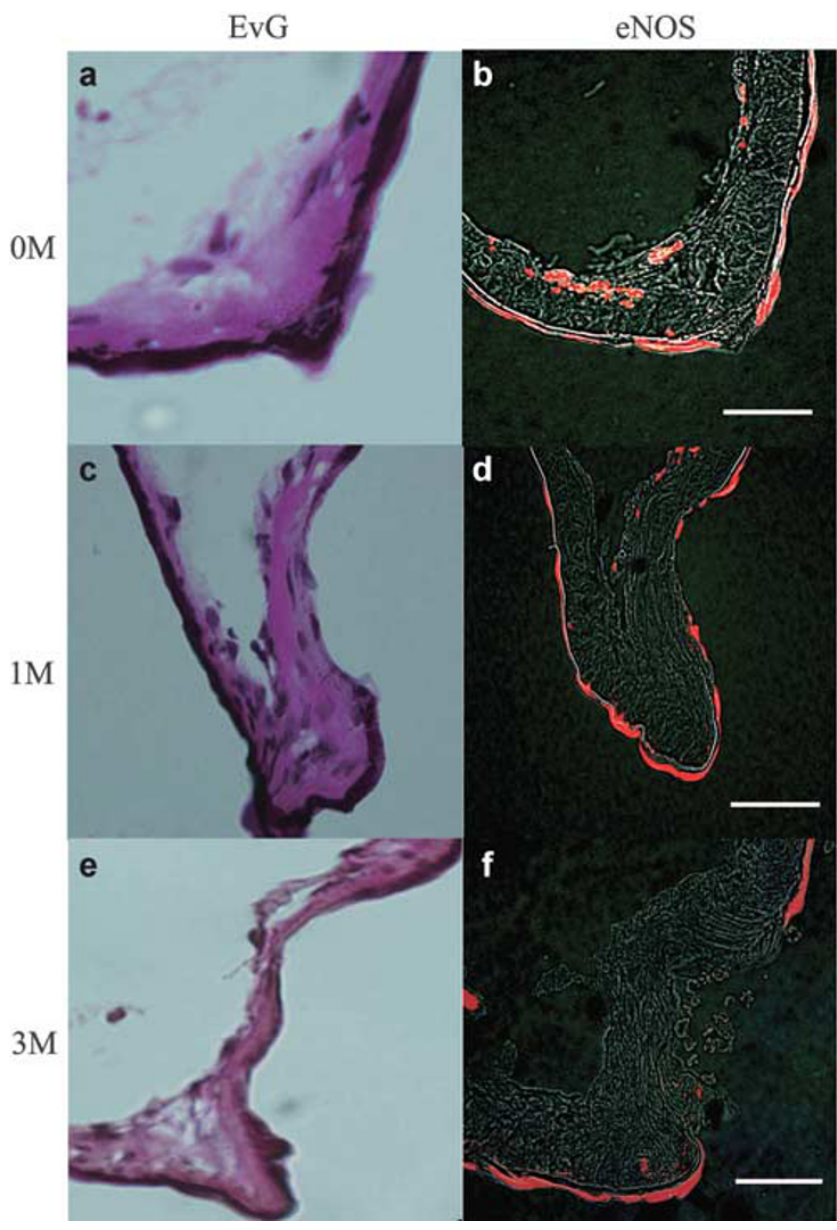

Figure 1 Expression of eNOS in experimentally induced CA walls of rats in situ by immunostaining. Elastica van Gieson (EvG) staining (a, c, e) and immunostaining for eNOS (red signal: $\mathbf{b}, \mathbf{d}, \mathbf{f}$ ) in CA walls of rats before (OM; $\mathbf{a}, \mathbf{b}), 1$ month $(1 \mathrm{M} ; \mathbf{c}, \mathbf{d})$ and 3 months $(3 \mathrm{M} ; \mathbf{e}, \mathbf{f})$ after CA induction. Serial sections of (a, $\mathbf{c}$ or $\mathbf{e})$ were used for immunostaining as shown in (b, $\mathbf{d}$ or $\mathbf{f})$, respectively. Square indicated the region of CA. Arrow shows the disruption of internal elastic lamina. $\mathrm{Bar}=20 \mu \mathrm{m}$.

These results combined together suggest that the expression of eNOS in endothelial cells is downregulated at the site of CA progression, although it was upregulated in whole circle of Willis.

\section{CA Formation in eNOS ${ }^{-1-}$ Mice}

To confirm the role of eNOS in CA formation, $\mathrm{eNOS}^{+/+}$ and $\mathrm{eNOS}^{-1-}$ mice underwent CA induction. CA formation at the right ACA/OA bifurcation was histopathologically evaluated 5 months after CA induction and classified into three categories as described above. There were no anatomic differences in the circle of Willis between $\mathrm{eNOS}^{-1-}$ and $\mathrm{eNOS}^{+/+}$mice (data not shown).

After 5 months of CA induction, 4 of $10 \mathrm{eNOS}^{-1-}$ mice (40\%) presented early aneurysmal changes and 2 (20\%) presented advanced aneurysms. In eNOS ${ }^{+/+}$mice, 10 of 18 mice $(56 \%)$ developed aneurysmal changes and $3(17 \%)$ 
a
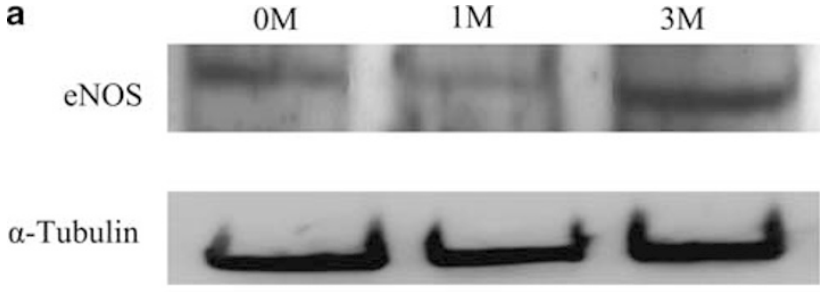

b

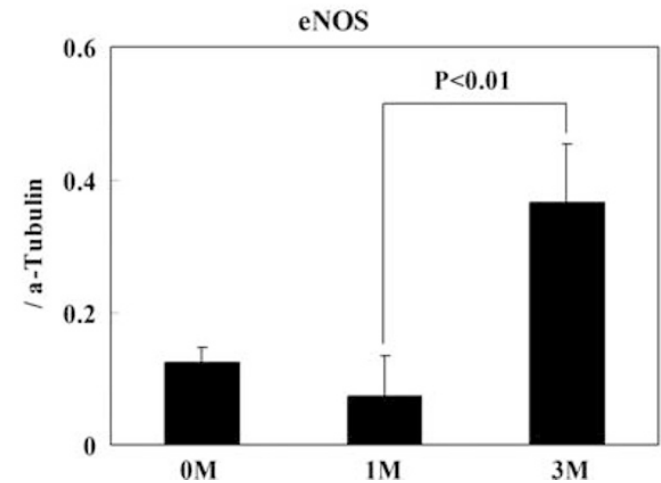

Figure 2 Expression of eNOS protein in the circle of Willis during CA formation by western blot analysis. (a) Representative image of western blot analysis for eNOS in rats from five independent experiments.

(b) Densitometric analysis $(n=5)$. $\alpha$-Tubulin served as an internal control. Before (OM), 1 month (1M) and 3 months ( $3 M)$ after CA induction.

developed advanced aneurysms. The incidence of all aneurysmal changes, including early aneurysmal changes and advanced aneurysms, was not statistically different (Figure 3a). eNOS deficiency is well known to increase systemic blood pressure. eNOS-deficient mice used in this study certainly showed the tendency of increased systemic blood pressure, but the differences did not reach statistically significant level $\left(\mathrm{eNOS}^{+1+}\right.$ mice: 91.5 (17.7) $\mathrm{mm} \mathrm{Hg}$ at 0 months, $n=10$; $128.2(8.9) \mathrm{mm} \mathrm{Hg}$ at 5 months, $n=10$; $\mathrm{eNOS}^{-1-}$ mice: 96.9 (14.2) $\mathrm{mm} \mathrm{Hg}$ at 0 months, $n=10 ; 155.2(30.7) \mathrm{mm} \mathrm{Hg}$ at 5 months, $n=9$; NOSS $^{-1-}$ versus eNOS ${ }^{+1+}$ mice: $P=0.096$; Figure $3 b)$.

\section{MCP-1 and VCAM-1 Expression and Subsequent Macrophage Infiltration in Experimentally Induced CA Walls of Mice}

Macrophages are the main inflammatory cells in CA walls and have a critical role in CA formation. ${ }^{22}$ To examine the chronic inflammation in CA walls, we assessed the macrophage infiltration and responsible molecules, VCAM-1 and MCP-1. VCAM-1 was the adhesion molecule responsible for transendothelial migration of macrophages and MCP-1 was a major chemoattractant for macrophages.

VCAM-1 expression was not detectable, and MCP-1 expression was only weakly detected in control arterial walls of both $\mathrm{eNOS}^{-1-}$ and eNOS ${ }^{+1+}$ mice (Figure 4a). These expressions were significantly elevated after CA induction, but there was no significant difference (Figure $4 a-c$ ). In immunostaining, the expression of MCP-1 and VCAM-1 a
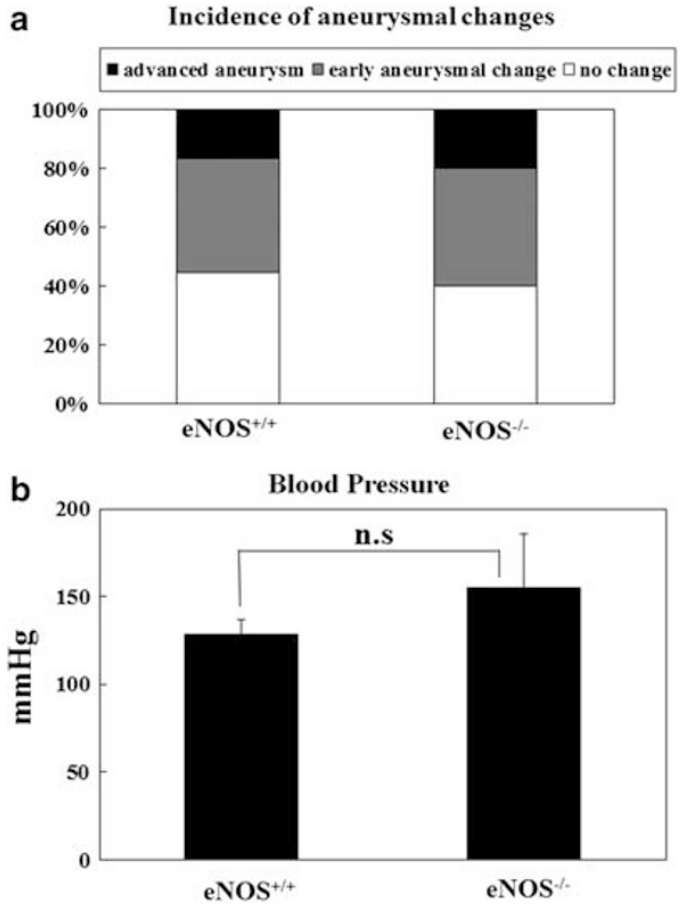

Figure 3 The effect of eNOS deficiency on CA formation. The incidence of CA formation in male eNOS ${ }^{+1+}$ and eNOS $^{-1-}$ mice (a) and systemic blood pressure (b) at 5 months after CA induction are shown; n.s., statistically not significant: $P>0.05$.

in CA walls of both mice was similar, consistent with the results of RT-PCR (Figure 4d-i). Macrophage infiltration in CA walls was also not different $\left(\mathrm{eNOS}^{-1-}: 4.2\right.$ (1.3) cells per $100 \mu \mathrm{m}^{2}, n=15$; $\mathrm{eNOS}^{+/+}: 4.2$ (2.5) cells per $100 \mu \mathrm{m}^{2}$, $n=10$; Figure $4 \mathrm{j})$.

These results combined together suggest that eNOS deficiency did not influence the chronic inflammation and resultant CA formation.

\section{Compensation of eNOS Deficiency by nNOS}

There are three types of NOS: eNOS, iNOS and nNOS.7 eNOS deficiency failed to influence CA formation. One of the possible mechanisms is that other NOS family members compensate for eNOS deficiency. To verify whether compensatory mechanisms were present, we examined the expression of nNOS and iNOS in the CA walls of $\mathrm{eNOS}^{-/-}$mice.

Immunostaining revealed that eNOS expression was certainly present in the CA walls of eNOS ${ }^{+l+}$ mice and was absent in eNOS ${ }^{-1-}$ mice (Figure $5 \mathrm{a}$ and $\mathrm{b}$ ). Both nNOS and iNOS were expressed in CA walls. Majority of both signals were not merged with signals of PECAM-1 (red signals in the figure), suggesting that the major expression site was the media of CA walls in both $\mathrm{eNOS}^{-1-}$ and eNOS ${ }^{+1+}$ mice. Signals of nNOS were increased in both the endothelial cells and the media of the CA walls of eNOS ${ }^{-1-}$ mice (Figure $5 \mathrm{c}$ and d). Expression of iNOS was also increased, but only 
a
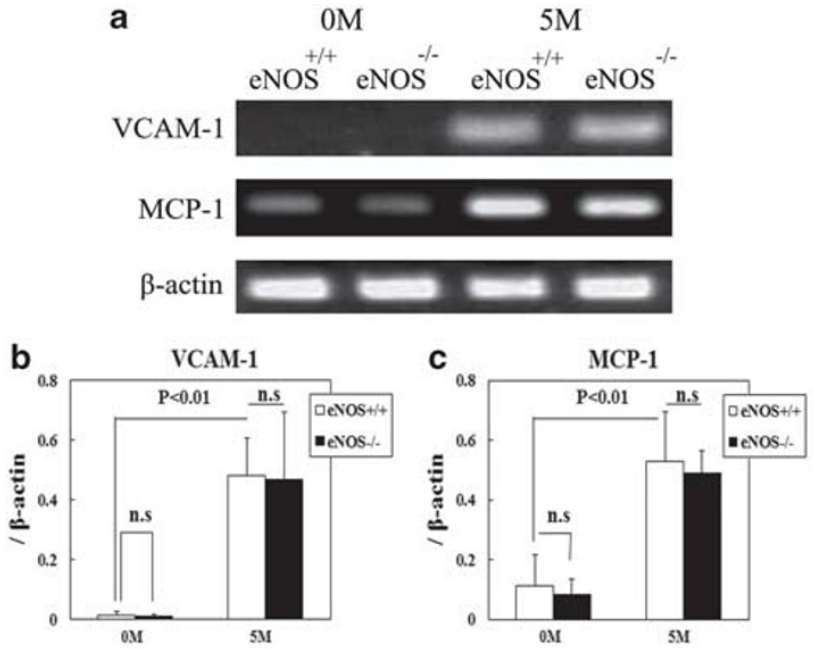

EvG
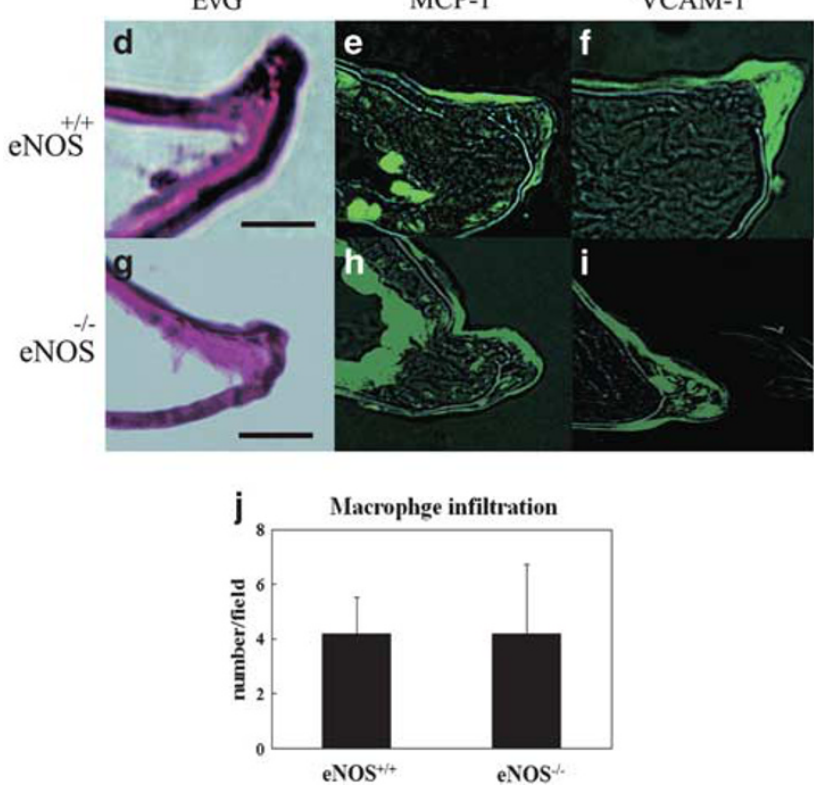

Figure 4 Expression of MCP-1 and VCAM-1 mRNA in the circle of Willis and subsequent macrophage infiltration in CA walls of eNOS ${ }^{-1-}$ mice. Representative image of RT-PCR analysis for MCP-1 and VCAM-1 (a) and densitometric analysis $(\mathbf{b}, \mathbf{c})$ from five independent experiments. $\beta$-Actin served as an internal control. Before (OM) and 5 months (5M) after CA induction; n.s., statistically not significant. EvG staining (d, g), immunostaining for MCP-1 (green signal: $\mathbf{e}, \mathbf{h}$ ) and VCAM-1 (green signal: $\mathbf{f}, \mathbf{i})$ in CA walls of eNOS ${ }^{+1+}(\mathbf{e}, \mathbf{f})$ and $\mathrm{eNOS}^{-1-}$ mice (h, i). Bar $=20 \mu \mathrm{m}$. The numbers of infiltrated macrophages in CA walls are shown in (j).

slightly, in the endothelial cell layer of CA walls (Figure 5e and f). In western blot analysis, nNOS expression was significantly higher in $\mathrm{eNOS}^{-1-}$ mice than in $\mathrm{eNOS}^{+/+}$mice $(P<0.01, n=5$ in each group $)$. On the contrary, iNOS expression was not different between both mice (Figure $5 \mathrm{~g}$ ). Like western blot analysis, RT-PCR revealed that in cerebral arteries without CA induction, the expression of nNOS was higher in $\mathrm{eNOS}^{-1-}$ mice than in eNOS ${ }^{+/+}$mice and that the expression of nNOS was significantly upregulated in
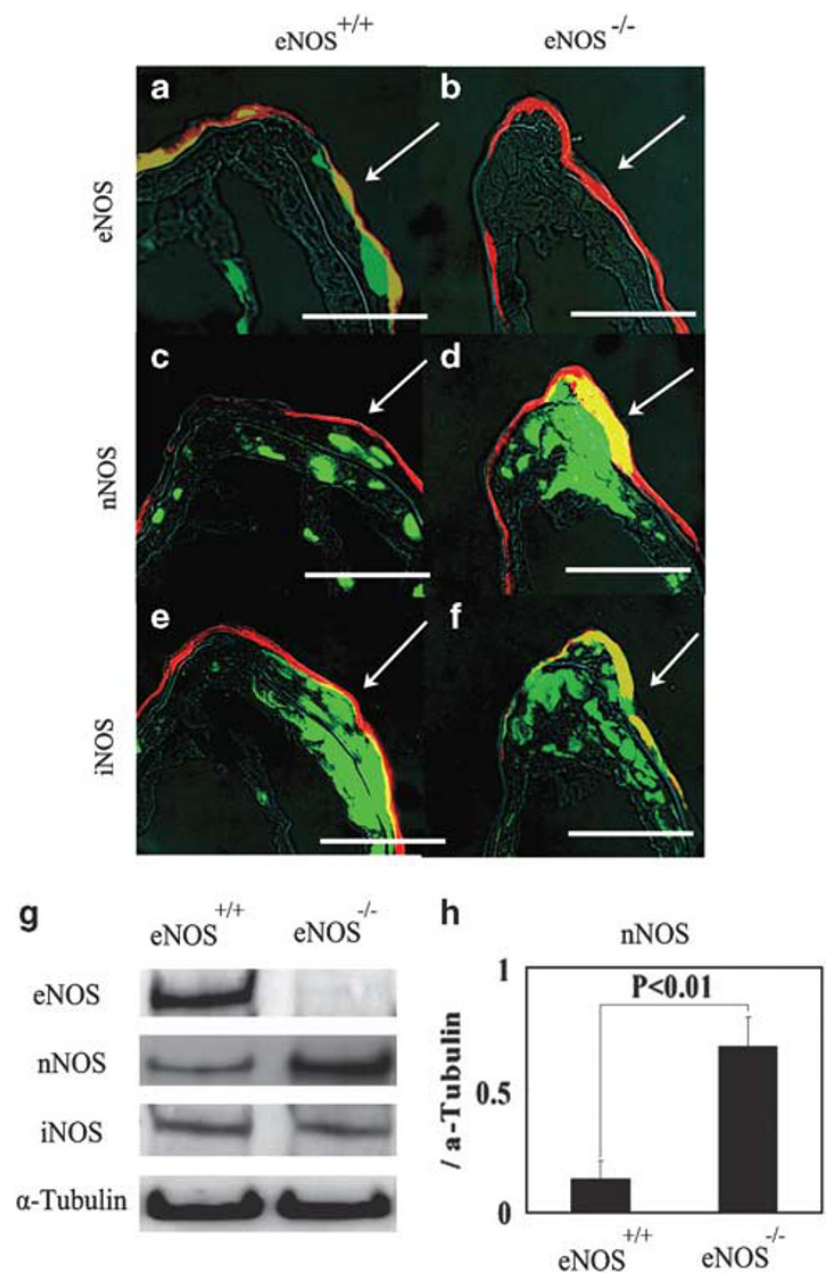

Figure 5 Expression of $\mathrm{nNOS}$ and iNOS in $\mathrm{eNOS}^{-1-}$ mice. Immunostaining for eNOS $(\mathbf{a}, \mathbf{b}), \mathrm{nNOS}(\mathbf{c}, \mathbf{d})$ and iNOS $(\mathbf{e}, \mathbf{f})$ (green signal) in CA walls of $\mathrm{eNOS}^{+I^{+}}(\mathbf{a}, \mathbf{c}, \mathbf{e})$ and $\mathrm{eNOS}^{-1-}(\mathbf{b}, \mathbf{d}, \mathbf{f})$ mice. Red signal indicates the expression of endothelial cell marker, PECAM-1. Bar $=30 \mu \mathrm{m}$. Arrows point to the CA walls. Representative images of western blot analysis for eNOS, nNOS and iNOS using the proteins from the circle of Willis from five independent experiments $(\mathbf{g})$ and densitometric analysis (h) are shown. $\alpha$-Tubulin served as an internal control.

$\mathrm{eNOS}^{-1-}$ mice compared with $\mathrm{eNOS}^{+/+}$mice after CA induction. Although iNOS expression was certainly upregulated in both $\mathrm{eNOS}^{-1-}$ and eNOS ${ }^{+1+}$ mice, there was no difference (Supplementary Figure 2).

These results combined together indicate the possibility that nNOS compensates for the effect of eNOS in cerebral arteries and CA walls of $\mathrm{eNOS}^{-1-}$ mice.

\section{CA Induction in $\mathrm{eNOS}^{-1-}$ nNOS $^{-1-}$ Mice}

To confirm the compensatory effect of nNOS in eNOS ${ }^{-1-}$ mice, we established the $\mathrm{eNOS}^{-1-} \mathrm{nNOS}^{-1-}$ mice (Figure 6a) and induced CA. All mice used in this experiment were 7-week-old male littermates.

nNOS deficiency did not influence systemic blood pressure $\left(\mathrm{eNOS}^{-1-} \mathrm{nNOS}^{+/+}\right.$mice: 155.2 (30.7) $\mathrm{mm} \mathrm{Hg}, n=10$; 
a

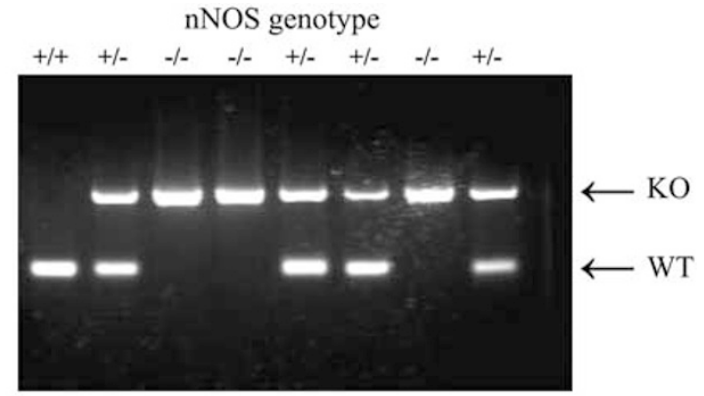

b Incidence of aneurysmal changes
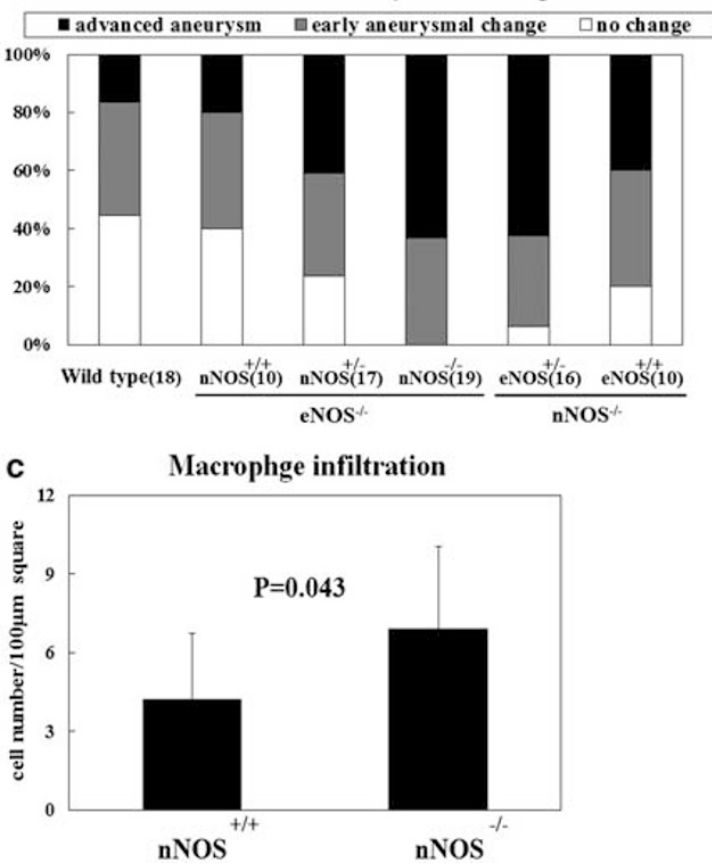

Figure 6 The effect of nNOS deletion on CA formation in eNOS ${ }^{-1-}$ mice. (a) A representative image of genotyping of the nNOS allele in eNOS ${ }^{-1-}$ mice. KO, knockout; WT, wild type. (b) The incidence of CA at 5 months after CA induction. The number of mice is shown in parentheses. (c) The number of macrophages infiltrated in CA walls of nNOS ${ }^{+/+} \mathrm{eNOS}^{-1-}$ and $\mathrm{nNOS}^{-1-} \mathrm{eNOS}^{-1-}$ mice.

eNOS $^{-1-}$ nNOS $^{-1-}$ mice: 142.7 (10.9) $\mathrm{mm} \mathrm{Hg}, \quad n=7$ ). In $\mathrm{eNOS}^{-1-} \mathrm{nNOS}^{+/-}$mice, the incidence of all aneurysmal changes was $76 \%$ ( 13 of 17 ), and $41 \%$ of mice (7 of 17) developed the advanced aneurysm. In eNOS ${ }^{-1-}$ nNOS $^{-1-}$ mice, the incidence of all aneurysmal changes was $100 \%$ (19 of 19) and $63 \%$ of mice (12 of 19) developed advanced aneurysms (Figure $6 \mathrm{~b}$ ). Through the deletion of nNOS in $\mathrm{eNOS}^{-1-}$ mice, the incidence of CA formation significantly increased in $\mathrm{eNOS}^{-1-} \mathrm{nNOS}^{-1-}$ mice compared with $\mathrm{eNOS}^{+1+} \mathrm{nNOS}^{+/+}$mice $(P<0.01$; Figure $6 \mathrm{~b})$. Furthermore, the incidence of advanced aneurysms also significantly increased through the deletion of nNOS with gene dosage effect $\left(\mathrm{eNOS}^{+/+} \mathrm{nNOS}^{+/+}\right.$mice, $\mathrm{eNOS}^{-1-} \mathrm{nNOS}^{+/+}$mice and $\mathrm{eNOS}^{-1-} \mathrm{nNOS}^{+/-}$mice versus $\mathrm{eNOS}^{-1-} \mathrm{nNOS}^{-1-}$ mice: $P<0.01, P<0.01$ and $P=0.040$, respectively, Figure $6 \mathrm{~b}$ ).
Notably, CA formation in $\mathrm{nNOS}^{-1-}$ mice (8 of 10) was not significantly different $(P=0.25)$ with observed in wild-type mice like $\mathrm{eNOS}^{-1-}$ mice, suggesting that eNOS and nNOS had the functional redundancy in cerebral arteries (Figure 6b).

Macrophage infiltration in CA walls was significantly higher in $\mathrm{eNOS}^{-1-} \mathrm{nNOS}^{-1-}$ mice than in $\mathrm{eNOS}^{-1-}$ $\mathrm{nNOS}^{+/+}$mice $\left(\mathrm{eNOS}^{-1-} \mathrm{nNOS}^{+/+}\right.$mice: 4.2 (2.5) cells per $100 \mu \mathrm{m}^{2}, n=10$; $\mathrm{eNOS}^{-1-} \mathrm{nNOS}^{-1-}$ mice: 6.9 (3.1) cells per $100 \mu \mathrm{m}^{2}, n=10 ; P=0.043$; Figure $6 \mathrm{c}$ ).

These results suggest that nNOS can compensate for the protective effect of eNOS on CA formation.

\section{DISCUSSION}

This study, in which we examined the role of eNOS in CA formation mainly by eNOS ${ }^{-1-}$ mice, yielded two important results: (1) eNOS deficiency is functionally compensated by nNOS in cerebral arteries and (2) eNOS and nNOS cooperatively have a protective role in the inflammation of cerebral arteries and suppress resultant CA formation. Our hypothesis about the contribution of eNOS and nNOS in CA formation from this study is that in CA walls, eNOS expression is downregulated because of the damage and hypofunction of endothelial cells in CA walls, probably by shear stress loaded. nNOS mainly produced by media contributed to the suppression of CA formation by compensating the eNOS downregulation in endothelial cells. However, at the late stage of CA formation, in which media of CA walls was also degenerated, nNOS expression may also be reduced, resulting in the CA formation as in double knockout mice.

Arterial walls are continuously loaded by circulating blood flow, which generates hemodynamic force. NO produced by NOS is one of the most important protective mechanisms against the continuous hemodynamic force. ${ }^{23}$ There are three members in the NOS family. Among them, eNOS is expressed in endothelial cells of arterial walls and most importantly contributes to the arterial dilatation through NO production. ${ }^{23}$ Because hemodynamic force with subsequent mechanical injury in arterial walls is the underlying common mechanism of various arterial diseases, eNOS is believed to have a protective effect on arterial diseases. In fact, many experimental findings have verified the protective role of eNOS against arterial diseases. For example, $\mathrm{eNOS}^{-1-}$ mice exhibit an increased incidence and severity of atherosclerotic lesions ${ }^{10}$ and AAA. ${ }^{10}$ In this study, we first hypothesized that eNOS $^{-1-}$ mice show an increased incidence of CA formation, like the results in atherosclerosis and AAA. ${ }^{10}$ Besides initial hypothesis, eNOS ${ }^{-1-}$ mice failed to exhibit the increased incidence of CA formation through the compensation of eNOS deficiency by nNOS upregulation, which is the other member of the NOS family (Figures 3-5). The functional compensation of eNOS deficiency by nNOS was confirmed by CA induction in eNOS ${ }^{-1-} \mathrm{nNOS}^{-1-}$ mice. These $\mathrm{eNOS}^{-1-} \mathrm{nNOS}^{-1-}$ mice demonstrated the remarkably increased incidence of CA formation through accelerating arterial inflammation assessed by infiltrated macrophages (Figure 6). 
nNOS and eNOS are both constitutively expressed in arterial walls, mainly in endothelial cells and nerves, ${ }^{24,25}$ respectively, and coordinately have a role in relieving hemodynamic force. Recent experimental studies have revealed that like eNOS, nNOS has a powerful protective role in various arterial diseases. ${ }^{8,26,27}$ In fact, nNOS is upregulatedly expressed in various kinds of cells, such as endothelial cells, smooth muscle cells and macrophages, and in lesions such as neointima in response to hemodynamic force and inflammation. ${ }^{8,27}$ Furthermore, nNOS ${ }^{-1-}$ mice exhibit the increased incidence of atherosclerotic lesions and neointimal formation. ${ }^{8,26}$ Recent studies have indicated that nNOS can compensate for the function of eNOS in $\mathrm{eNOS}^{-1-}$ mice. For example, the expression of nNOS is upregulated in the brain, ${ }^{28}$ the muscles ${ }^{28}$ and the smooth muscle cells of coronary arteries $^{29}$ of eNOS ${ }^{-1-}$ mice at the protein level. The distribution of nNOS in the retina of $\mathrm{eNOS}^{-1-}$ mice is stronger in the vasculature of deep layer, where eNOS is abundantly expressed in wild-type mice than in wild-type mice. ${ }^{30}$ Vasodilatation of coronary arteries by eNOS-producing $\mathrm{NO}$ is partially compensated for by nNOS in eNOS ${ }^{-1-}$ mice. $^{31}$ In CA walls of $\mathrm{eNOS}^{-1-}$ mice, the expression of nNOS is upregulated mainly in smooth muscle cells and endothelial cells, as shown in the studies of coronary arteries. In various pathological settings, in which the function of endothelial cells is suppressed through the damage of cells, nNOS may functionally compensate eNOS downregulation as the safety mechanisms against diseases. We should consider the possibility that the functional compensation can be achieved by the increased enzymatic activity of NOS not by the changes of expression itself. However, unfortunately, we have no suitable methods to assess the enzymatic activity of NOS in vivo, and hence there is certainly a limitation in this study. Interestingly, the compensational effects of nNOS have been observed only in small- to middlesized arteries but not in the aorta. These results may suggest a functional difference in the regulation of NOS expression according to the size of arteries and a similarity between cerebral arteries and coronary arteries. Furthermore, in cerebral arteries, we have first confirmed that nNOS can functionally compensate for the deficiency of eNOS.

In AAA, the polymorphism of eNOS (G894T) is reportedly associated with a mild risk of AAA. ${ }^{32}$ In contrast, some recent studies on the polymorphism of eNOS have failed to demonstrate the positive relation with CA formation or rupture. ${ }^{33}$ These results may suggest that the dysfunction of eNOS can be compensated for with nNOS upregulation in cerebral arteries unlike in the aorta and further strengthen the hypothesis that there is a functional difference in NOS regulation.

Previously, Abruzzo et $a l^{34}$ demonstrated that eNOS deficiency contributed to CA formation, unlike the result of our study. However, there were some critical differences between their study and this study. Abruzzo et $a l^{34}$ induced CA only by carotid ligation and observed longer period than our model. We could induce CA in wild-type mice as shown in this study, whereas they failed to induce CA in wild-type mice, suggesting the critical difference of two models. Furthermore, we and they used mice with different gender. Sex hormone, especially estrogen, is well known to have a vasoprotective and anti-inflammatory effect. ${ }^{35}$ As inflammation in arterial walls greatly influence CA formation, ${ }^{14,36}$ estrogen with anti-inflammatory effect may have significantly participated in the pathogenesis of CA, resulting in the sex difference of CA formation. Furthermore, we demonstrated that nNOS compensated the eNOS deficiency in cerebral arteries. There are many reports indicating that estrogen regulates nNOS expression in both up- and down-regulated manner. ${ }^{37-39}$ Combined with the above discussion, sex hormone, especially estrogen, has a considerable impact on CA formation and can greatly influence CA formation. This is because there may be a different result in CA formation of eNOS-deficient mice with different sex. Indeed, a previous study using rat CA model demonstrated that sex hormone significantly contributed to CA formation. ${ }^{40}$

Another inducible type of NOS whose expression is activated by various stimuli is iNOS. Both eNOS and nNOS constitutively produce a small amount of $\mathrm{NO}$ and have their role under basal conditions. On the contrary, iNOS produces a large amount of NO in response to inflammatory stimuli and contributes to various inflammatory diseases. ${ }^{7,27}$ In CA, iNOS contributes to CA progression by inducing apoptosis in smooth muscle cells of CA walls. ${ }^{14,41}$ Furthermore, iNOS also contributes to the chronic inflammation by the production of reactive oxygen species in CA walls. ${ }^{42}$ Combined with the results from this study, the NOS family has a close relation with CA formation. Both eNOS and nNOS are constitutively expressed in cerebral arteries and have the protective role in CA formation. On the contrary, the expression of iNOS is induced by inflammatory stimuli and contributes to the progression of CA.

Our data suggest that eNOS and nNOS have functional redundancy in cerebral arteries and have the coordinate role as key molecules for preventing the formation of CAs by reducing hemodynamic stress to arterial walls. At present, CA is managed by surgical clipping or coil embolization. There is no medical treatment to suppress the progression and rupture of CAs. In the future, it might be possible to modulate eNOS or nNOS expression to inhibit CA progression and thereby reduce the risk of subarachnoid hemorrhage.

Supplementary Information accompanies the paper on the Laboratory Investigation website (http://www.laboratoryinvestigation.org)

\section{ACKNOWLEDGEMENT}

This work was supported by Grants-in-Aid for Scientific Research (numbers 20791008 and 19890112) from the Ministry of Education, Science and Culture of Japan.

\section{DISCLOSURE/CONFLICT OF INTEREST}

The authors declare no conflict of interest. 
1. van Gijn J, Kerr RS, Rinkel GJ. Subarachnoid haemorrhage. Lancet 2007;369:306-318.

2. Wiebers DO, Whisnant JP, Huston III J, et al. Unruptured intracranial aneurysms: natural history, clinical outcome, and risks of surgical and endovascular treatment. Lancet 2003;362:103-110.

3. Wermer MJ, Kool H, Albrecht KW, et al. Subarachnoid hemorrhage treated with clipping: long-term effects on employment, relationships, personality, and mood. Neurosurgery 2007;60:91-97.

4. Castro MA, Putman CM, Sheridan MJ, et al. Hemodynamic patterns of anterior communicating artery aneurysms: a possible association with rupture. AJNR Am J Neuroradiol 2009;30:297-302.

5. Hashimoto $\mathrm{N}$, Handa $\mathrm{H}, \mathrm{Nagata} \mathrm{l}$, et al. Experimentally induced cerebral aneurysms in rats: Part V. Relation of hemodynamics in the circle of Willis to formation of aneurysms. Surg Neurol 1980;13:41-45.

6. Ballermann BJ, Dardik A, Eng E, et al. Shear stress and the endothelium. Kidney Int Suppl 1998;67:S100-S108.

7. Andrew PJ, Mayer B. Enzymatic function of nitric oxide synthases. Cardiovasc Res 1999;43:521-531.

8. Morishita T, Tsutsui M, Shimokawa $H$, et al. Vasculoprotective roles of neuronal nitric oxide synthase. FASEB J 2002;16:1994-1996.

9. Chen J, Kuhlencordt PJ, Astern J, et al. Hypertension does not account for the accelerated atherosclerosis and development of aneurysms in male apolipoprotein e/endothelial nitric oxide synthase double knockout mice. Circulation 2001;104:2391-2394.

10. Kuhlencordt PJ, Gyurko R, Han F, et al. Accelerated atherosclerosis, aortic aneurysm formation, and ischemic heart disease in apolipoprotein E/endothelial nitric oxide synthase double-knockout mice. Circulation 2001;104:448-454.

11. Hashimoto N, Handa H, Hazama F. Experimentally induced cerebra aneurysms in rats. Surg Neurol 1978;10:3-8.

12. Morimoto M, Miyamoto S, Mizoguchi A, et al. Mouse model of cerebra aneurysm: experimental induction by renal hypertension and local hemodynamic changes. Stroke 2002;33:1911-1915.

13. Nagata I, Handa H, Hashimoto N. Experimentally induced cerebral aneurysms in rats: part IV-cerebral angiography. Surg Neurol 1979;12:419-424.

14. Aoki T, Kataoka H, Shimamura M, et al. NF-kappaB is a key mediator of cerebral aneurysm formation. Circulation 2007;116:2830-2840.

15. Hashimoto N. Experimental-model for producing cerebral aneurysms. J Neurosurg 1987;66:634.

16. Hashimoto $\mathrm{N}$, Handa $\mathrm{H}$, Hazama F. Experimentally induced cerebral aneurysms in rats. 3. Pathology. Surg Neurol 1979;11:299-304.

17. Aoki T, Kataoka H, Ishibashi R, et al. Reduced collagen biosynthesis is the hallmark of cerebral aneurysm: contribution of interleukin-1beta and nuclear factor-kappaB. Arterioscler Thromb Vasc Biol 2009;29:1080-1086.

18. Shesely EG, Maeda N, Kim HS, et al. Elevated blood pressures in mice lacking endothelial nitric oxide synthase. Proc Natl Acad Sci USA 1996;93:13176-13181.

19. Inci S, Spetzler RF. Intracranial aneurysms and arterial hypertension: a review and hypothesis. Surg Neurol 2000;53:530-540.

20. Kim C, Cervos-Navarro J, Kikuchi $\mathrm{H}$, et al. Alterations in cerebral vessels in experimental animals and their possible relationship to the development of aneurysms. Surg Neurol 1992;38:331-337.

21. Kim C, Cervos-Navarro J, Kikuchi H, et al. Degenerative changes in the internal elastic lamina relating to the development of saccular cerebral aneurysms in rats. Acta Neurochir (Wien) 1993;121:76-81.

22. Aoki T, Kataoka $\mathrm{H}$, Ishibashi $\mathrm{R}$, et al. Impact of monocyte chemoattractant protein-1 deficiency on cerebral aneurysm formation. Stroke 2009;40:942-951.
23. Gimbrone Jr MA. Endothelial dysfunction, hemodynamic forces, and atherosclerosis. Thromb Haemost 1999;82:722-726.

24. Tsutsui M. Neuronal nitric oxide synthase as a novel anti-atherogenic factor. J Atheroscler Thromb 2004;11:41-48.

25. Nangle MR, Cotter MA, Cameron NE. An in vitro investigation of aorta and corpus cavernosum from eNOS and nNOS gene-deficient mice. Pflugers Arch 2004;448:139-145.

26. Kuhlencordt PJ, Hotten S, Schodel J, et al. Atheroprotective effects of neuronal nitric oxide synthase in apolipoprotein e knockout mice. Arterioscler Thromb Vasc Biol 2006;26:1539-1544

27. Tsutsui $M$, Shimokawa $H$, Otsuji $Y$, et al. Nitric oxide synthases and cardiovascular diseases: insights from genetically modified mice. Circ J 2009;73:986-993.

28. Sanz MJ, Hickey MJ, Johnston B, et al. Neuronal nitric oxide synthase (NOS) regulates leukocyte-endothelial cell interactions in endothelial NOS deficient mice. Br J Pharmacol 2001;134:305-312.

29. Talukder MA, Fujiki T, Morikawa K, et al. Up-regulated neuronal nitric oxide synthase compensates coronary flow response to bradykinin in endothelial nitric oxide synthase-deficient mice. J Cardiovasc Pharmacol 2004;44:437-445.

30. Al-Shabrawey M, El-Remessy A, Gu X, et al. Normal vascular development in mice deficient in endothelial NO synthase: possible role of neuronal NO synthase. Mol Vis 2003;9:549-558.

31. Huang A, Sun D, Shesely EG, et al. Neuronal NOS-dependent dilation to flow in coronary arteries of male eNOS-KO mice. Am J Physiol Heart Circ Physiol 2002;282:H429-H436.

32. Fatini C, Sofi F, Sticchi E, et al. eNOS G894T polymorphism as a mild predisposing factor for abdominal aortic aneurysm. J Vasc Surg 2005;42:415-419.

33. Akagawa $\mathrm{H}$, Kasuya $\mathrm{H}$, Onda $\mathrm{H}$, et al. Influence of endothelial nitric oxide synthase T-786C single nucleotide polymorphism on aneurysm size. J Neurosurg 2005;102:68-71.

34. Abruzzo T, Kendler A, Apkarian $\mathrm{R}$, et al. Cerebral aneurysm formation in nitric oxide synthase-3 knockout mice. Curr Neurovasc Res 2007;4: 161-169.

35. Xing D, Nozell S, Chen YF, et al. Estrogen and mechanisms of vascular protection. Arterioscler Thromb Vasc Biol 2009;29:289-295.

36. Kataoka H, Aoki T. Molecular basis for the development of intracranial aneurysm. Expert Rev Neurother 2010;10:173-187.

37. Game X, Allard J, Escourrou G, et al. Estradiol increases urethral tone through the local inhibition of neuronal nitric oxide synthase expression. Am J Physiol Regul Integr Comp Physiol 2008;294: R851-R857

38. Garcia-Duran $M$, de $T$, az-Recasens $J$, et al. Estrogen stimulates neuronal nitric oxide synthase protein expression in human neutrophils. Circ Res 1999;85:1020-1026.

39. Sica M, Martini M, Viglietti-Panzica $C$, et al. Estrous cycle influences the expression of neuronal nitric oxide synthase in the hypothalamus and limbic system of female mice. BMC Neurosci 2009;10:78.

40. Jamous MA, Nagahiro S, Kitazato KT, et al. Role of estrogen deficiency in the formation and progression of cerebral aneurysms. Part II: experimental study of the effects of hormone replacement therapy in rats. J Neurosurg 2005;103:1052-1057.

41. Sadamasa N, Nozaki K, Hashimoto N. Disruption of gene for inducible nitric oxide synthase reduces progression of cerebral aneurysms. Stroke 2003;34:2980-2984.

42. Aoki $T$, Nishimura $M$, Kataoka $H$, et al. Reactive oxygen species modulate growth of cerebral aneurysms: a study using the free radical scavenger edaravone and p47phox(-/-) mice. Lab Invest 2009;89: 730-741. 